OPEN ACCESS

Edited by:

Fei Gao,

Zhejiang University, China

Reviewed by:

Weiren Zhu,

Shanghai Jiao Tong University, China Zuojia Wang,

Shandong University, China

${ }^{*}$ Correspondence:

Hong Su

hsu@szu.edu.cn

Huawei Liang

hwliang@szu.edu.cn

Specialty section:

This article was submitted to Optics and Photonics,

a section of the journal

Frontiers in Physics

Received: 16 March 2020

Accepted: 23 July 2020

Published: 04 September 2020

Citation:

Li J, Ning T, Zhang M, Li IL, Su H and Liang H (2020) Realization of Terahertz Wavefront Manipulation Using Transmission-Type Dielectric Metasurfaces. Front. Phys. 8:349 doi: 10.3389/fphy.2020.00349

\section{Realization of Terahertz Wavefront Manipulation Using Transmission-Type Dielectric Metasurfaces}

\author{
Jiaqi Li ${ }^{1}$, Tingyin Ning ${ }^{2}$, Min Zhang ${ }^{1}$, Ireng Ling $\mathrm{Li}^{1}$, Hong Su ${ }^{1 *}$ and Huawei Liang ${ }^{1 *}$ \\ ${ }^{1}$ Shenzhen Key Laboratory of Laser Engineering, College of Physics and Optoelectronic Engineering, Shenzhen University, \\ Shenzhen, China, ${ }^{2}$ Shandong Provincial Engineering and Technical Center of Light Manipulations \& Shandong Provincial Key \\ Laboratory of Optics and Photonic Device, School of Physics and Electronics, Shandong Normal University, Jinan, China
}

Metasurfaces, composed of an array of subwavelength artificial structures, have attracted great interest, owing to their high ability in locally manipulating the wavefront of electromagnetic waves. Here, we propose a dielectric metasurface based on a fused silica resonator, consisting of a rectangular-shaped bar placed in the center of a cross net-shaped structure, to manipulate the wavefront of terahertz waves. As proof of concept, several transmission-type devices for spatial modulation are designed at the target frequency of $0.14 \mathrm{THz}$, including on-axis and off-axis focusing, generation of a non-diffracting Bessel beam, and multi-focus lens. The simulated efficiencies range from 45 to $62 \%$. This novel approach for manipulating $\mathrm{THz}$ wavefronts can be also used for information storage and other phase-related techniques in the rapid development of $\mathrm{THz}$ applications.

Keywords: metasurface, THz wave, spatial modulation, Bessel beam, multi-focus lens

\section{INTRODUCTION}

The traditional electromagnetic (EM) devices are designed to realize the control functionalities by adjusting the physical geometrical shape and the component material [1]. Recently, metasurfaces, made of a large number of subwavelength structures, have performed very well to locally manipulate the wavefront of EM waves. Many EM controls have achieved by metasurfaces, including ultrathin planar lenses [2-4], multi-focal devices [5-9], vortex beam [10-12], and various holography $[13,14]$. With the development of terahertz (THz) technology, highly efficient, compact functional devices are required, which can be provided by dielectric metasurfaces.

In this paper, we propose a transmission-type, fused silica metasurface to manipulate $\mathrm{THz}$ wavefront. Different from previous dielectric pillars on substrate $[10,11,15]$ or cross shaped restorers [16-19], the metasurface unit cell consists of a cross net shaped structure with a rectangular pillar placed in the center. The weak coupling of each basic unit is formed by the high refractive index difference between the fused silica and the surrounding air, which concentrates the confined energy within the central structure. Compared with dielectric pillars, the proposed metasurface have more adjustable unit structural parameters. Moreover, the metasurface reduces the difficulty in fabricating because each of the basic unit is reciprocally freestanding by cross shaped restorers without substrates. Through this work, on-axis and off-axis focusing, generation of a non-diffracting Bessel beam, and multi-spot focusing are demonstrated. The total control 
efficiency could be as high as $62 \%$ in numerical simulation. These advantages of the proposed metasurface will enrich $\mathrm{THz}$ functional devices, and provide a novel way for the design of multifunctional miniaturized devices.

\section{RESULTS AND DISCUSSION}

Figure 1A schematically shows the metasurface structure and the inset shows the details of geometry basic unit cell. Both the width $\boldsymbol{a}$ and length $\boldsymbol{b}$ are in the range of $0.3-1.9 \mathrm{~mm}$, respectively. The width of the cross net shaped structure $\boldsymbol{w}$ is set to $0.3 \mathrm{~mm}$. The period $\boldsymbol{T}$ and thickness $\boldsymbol{h}$ are $2 \mathrm{~mm}$ and $3.6 \mathrm{~mm}$, respectively. The amplitude transmission and corresponding phase delay for $x$-polarized incident waves are calculated using a commercially available software package COMSOL Multiphysics (Figures 1B,C). The phase shift can cover the range of $0-$ $360^{\circ}$, which facilitates the wave control. Eight unit cells are selected as $(a, b)=(0.8,1.6 \mathrm{~mm}),(0.58,1.5 \mathrm{~mm}),(0.54,1 \mathrm{~mm})$, $(0.35,0.35 \mathrm{~mm}),(1.9,1.86 \mathrm{~mm}),(1.65,1.75 \mathrm{~mm}),(1.25,1.7 \mathrm{~mm})$, $(0.95,1.65 \mathrm{~mm})$ for fully covering the phase range with an interval of $45^{\circ}$, as shown in Figure 1D. The average amplitude transmittance of the unit cells is $\sim 93 \%$. To demonstrate modulation ability of the proposed metasurface, several $\mathrm{THz}$ functional devices are designed based on the eight unit cells.

We first demonstrate on-axis and off-axis focusings achieved using the proposed metasurface. The phase distribution on the metasurface for realizing this focusing can be described as: $\varphi(x)=\frac{2 \pi}{\lambda_{0}}\left(\sqrt{\left(x-x_{0}\right)^{2}+L^{2}}-L\right)$, where $\lambda_{0}$ for free space, and $x$ and $x_{0}$ are the position coordinates of basic units and the focal spot, respectively, and $L$ is the focal length. The obtained phase profiles are quantized into eight values, ranging from 0 to $360^{\circ}$. The eight basic units are placed on the corresponding positions. When $x$-polarized $\mathrm{THz}$ waves are normally incident on the designed metasurface device, the metasurface modifies the wavefront. The transmitted waves are then focused into an on-axis spot with the propagation distance $L=5 \mathrm{~cm}$, as shown in Figure 2A. The control efficiency is defined as the ratio of the power of $E_{x}$ component on the focal plane to that of the incident wave. The on-axis focusing efficiency is $60 \%$, which is much higher than the single-layer plasmonic metasurfaces [20].
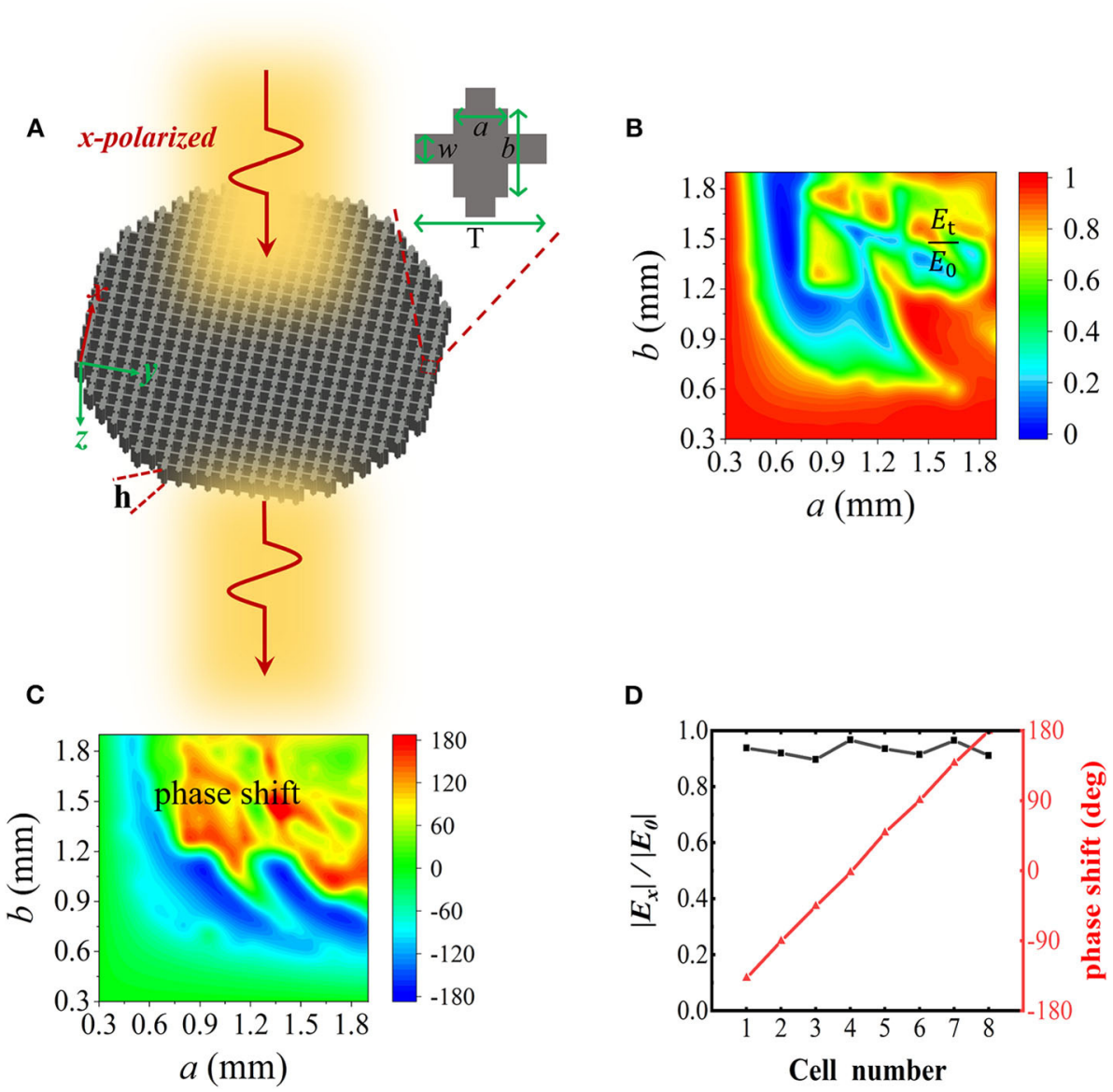

FIGURE 1 | Proposed dielectric metasurface and the control characteristics of metasurface unit cells. (A) Schematic of the proposed metasurface. The inset shows a basic unit cell of the metasurface. (B,C) Amplitude transmission and corresponding phase shift, respectively, of $x$-polarized wave with a frequency of 0.14 THz. (D) Phase shift and amplitude transmission for the selected eight unit cells. 


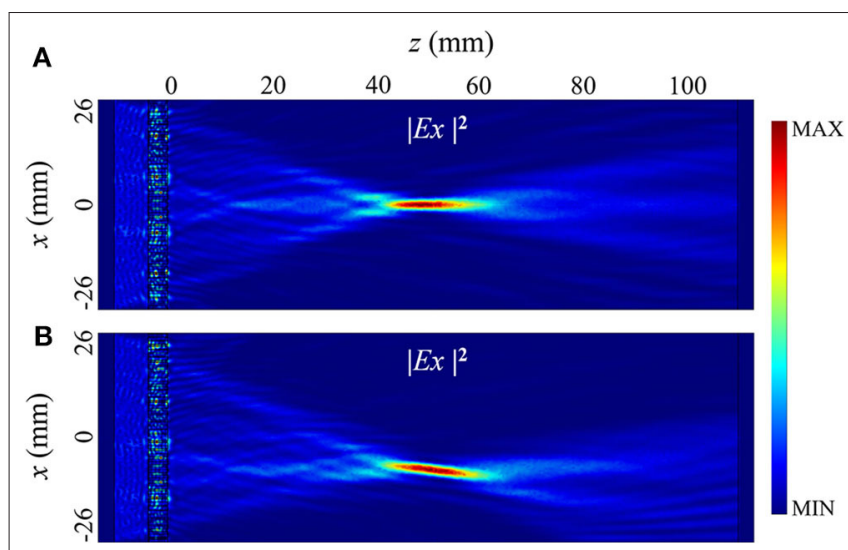

FIGURE 2 | Simulated intensity distributions of (A) on-axis and (B) off-axis focusings with a focal length $L=5 \mathrm{~cm}$.

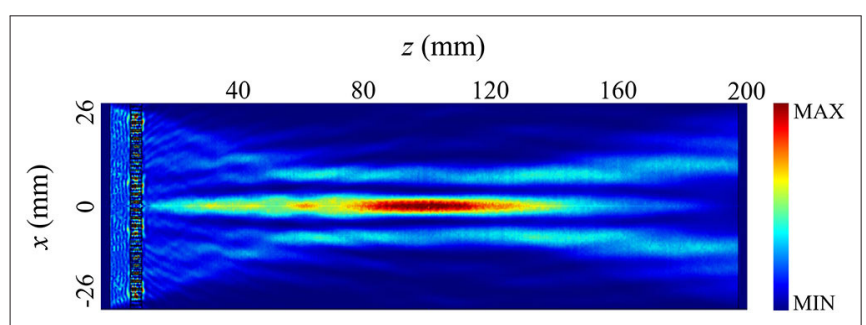

FIGURE 3 | Simulated intensity distribution of generation of a non-diffracting Bessel beam.

Furthermore, an off-axis focus lens with the same focal length $(L=5 \mathrm{~cm})$ is designed. The off-axis distance is $8 \mathrm{~mm}$, as shown by the simulated results in Figure 2B. The simulated control efficiency is $62 \%$.

Furthermore, we demonstrate generation of a non-diffracting Bessel beam using the dielectric metasurface. The phase distribution on the metasurface for generating a Bessel beam can be described as: $\varphi(x)=C \frac{2 \pi}{\lambda_{0}}|x|$, where $C$ is a coefficient. When $x$-polarized waves are normally incident on the metasurface with a width of $52 \mathrm{~mm}$, the arranged unit cells transform the wavefront into an axisymmetrical slope shape, so that a Bessel beam can be formed by the wave interference [21]. The simulated intensity distribution of a one-dimensional Bessel beam is shown in Figure 3. The control efficiency is $45 \%$. Compared with the focusing in Figure 2A, the focal depth of the Bessel beam is much longer and the beam width changes much more slowly along the long propagation direction, which are consistent with the typical characteristics of the non-diffractive Bessel beam.

To demonstrate the versatile control of the proposed metasurface, a one-dimensional multi-spot focusing lens (MSFL) is designed. The phase distributions for obtaining one-dimensional double-spot and tri-spot focusing are calculated, respectively, using the Gerchberg-Saxton (GS) retrieval algorithm [22] on the basis of Fresnel diffraction. In the simulations using the COMSOL Multiphysics, the required phase distributions can be obtained by arranging the aforementioned

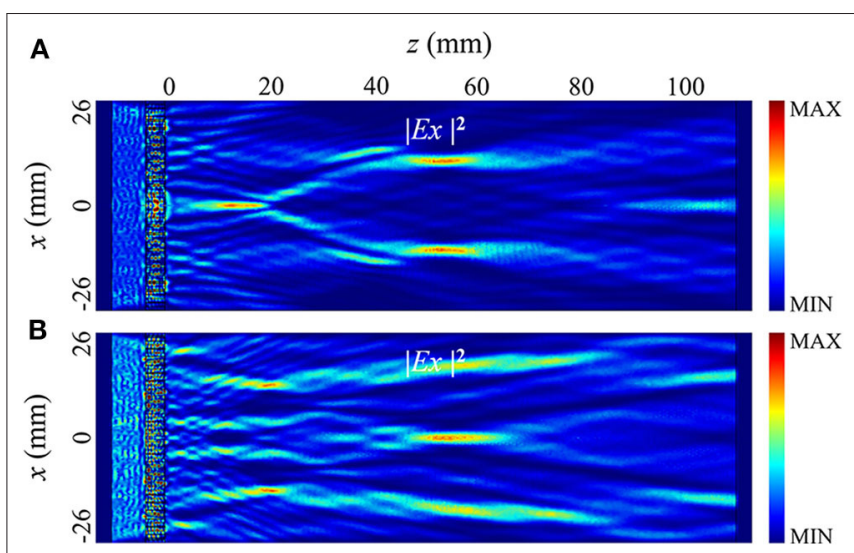

FIGURE 4 | Simulated intensity distributions of $(\mathbf{A}, \mathbf{B})$ double-spot and tri-spot focusing, respectively, with a focal length of $5 \mathrm{~cm}$.

unit cells in the one-dimensional metasurface with a width of $52 \mathrm{~mm}$. Then the double-spot and tri-spot focusing are formed by the wave interference, respectively. The simulated intensity distribution of one-dimensional double-spot focusing is shown in Figure 4A (off-axis $\pm 11 \mathrm{~mm}$ ). The control efficiencies for the two spots are 25 and $24 \%$ respectively. Thus, the total control efficiency is $49 \%$. Tri-spot focusing with the same focal length is also demonstrated, as shown in Figure 4B (on-axis and off-axis $\pm 17 \mathrm{~mm}$ ). The efficiency for each is 19,18 , and $18 \%$, and thus the total control efficiency is $55 \%$. The simulated field distributions on the focal plane are consistent with the predesigned results.

\section{CONCLUSION}

In summary, a dielectric metasurface based on a fused silica resonator, consisting of a cross net shaped structure with a rectangular pillar placed in the center, is proposed to manipulate $\mathrm{THz}$ waves. As a proof of concept, several transmission-type devices for $\mathrm{THz}$ spatial modulation are designed, including onaxis and off-axis focusing, generation of a non-diffracting Bessel beam, and multi-spot focusing. The simulated control efficiencies range from 45 to $62 \%$. The versatile control with high efficiency makes the metasurface valuable for the practical applications in $\mathrm{THz}$ communications and imaging.

\section{METHOD SECTION}

All simulations were using a commercial finite element simulation software COMSOL multiphysics. The refractive index of fused silica at the target frequency of $0.14 \mathrm{THz}$ is 1.95 . The perfect matching layers (PML) with a thickness of $3 \mathrm{~mm}$ were used along $z$-direction. The periodic boundary conditions were used in both $x$ - and $y$-directions to simulate the control characteristics of basic unit cells. In the simulations of functional devices, the scattering and periodic boundary conditions were used along the $x$ - and $y$-directions, respectively. 


\section{DATA AVAILABILITY STATEMENT}

The original contributions presented in the study are included in the article/supplementary materials, further inquiries can be directed to the corresponding author/s.

\section{AUTHOR CONTRIBUTIONS}

JL and HL proposed the idea and conceived and performed the simulations. TN designed the TMFL. MZ designed the Bessel

\section{REFERENCES}

1. Thompson KP, Rolland JP. Freeform optical surfaces: a revolution in imaging optical design. Opt Photonics News. (2012) 23:30-5. doi: 10.1364/opn.23.6.000030

2. Gao S, Park CS, Lee SS, Choi DY. A highly efficient bifunctional dielectric metasurface enabling polarization-tuned focusing and deflection for visible light. Adv Opt Mater. (2019) 7:1801337. doi: 10.1002/adom.201801337

3. Kamali SM, Arbabi E, Arbabi A, Horie Y, Faraon A. Highly tunable elastic dielectric metasurface lenses. Laser Photonics Rev. (2016) 10:1002-8. doi: 10.1002/lpor.201600144

4. Lin D, Fan P, Hasman E, Brongersma ML. Dielectric gradient metasurface optical elements. Science. (2014) 345:298-302. doi: 10.1126/science.1253213

5. Chen X, Zhang Y, Huang L, Zhang S. Ultrathin metasurface laser beam shaper. Adv Opt Mater. (2014) 2:978-82. doi: 10.1002/adom.201400186

6. Ding X, Wang Z, Zhang K, Gu X, Wu Q, Fu J, editors. Multi-focus imaging utilizing huygens metasurface. In: IEEE 2018 48th European Microwave Conference (EuMC). Madrid (2018). doi: 10.23919/eumc.2018.8541382

7. He J, Ye J, Wang X, Kan Q, Zhang Y. A broadband terahertz ultrathin multi-focus lens. Sci Rep. (2016) 6:28800. doi: 10.1038/srep28800

8. Wang Y, Guan C, Ding X, Zhang K, Ratni B, Burokur SN, et al. Multi-focus hologram utilizing Pancharatnam-Berry phase elements based metamirror. Opt Lett. (2019) 44:2189-92. doi: 10.1364/OL.44.002189

9. Zhu L, Xiong Z, Yu W, Tian X, Kong Y, Liu C, et al. Polarizationcontrolled tunable multi-focal plasmonic lens. Plasmonics. (2017) 12:33-8. doi: 10.1007/s11468-016-0225-2

10. Yang Y, Wang W, Moitra P, Kravchenko II, Briggs DP, Valentine J. Dielectric meta-reflectarray for broadband linear polarization conversion and optical vortex generation. Nano Lett. (2014) 14:1394-9. doi: 10.1021/nl4044482

11. Zhang $\mathrm{H}$, Zhang $\mathrm{X}, \mathrm{Xu}$ Q, Tian $\mathrm{C}$, Wang $\mathrm{Q}, \mathrm{Xu} \mathrm{Y}$, et al. High-efficiency dielectric metasurfaces for polarization-dependent terahertz wavefront manipulation. Adv Opt Mater. (2018) 6:1700773. doi: 10.1002/adom.201700773

12. Ou K, Li G, Li T, Yang H, Yu F, Chen J, et al. High efficiency focusing vortex generation and detection with polarization-insensitive dielectric metasurfaces. Nanoscale. (2018) 10:19154-61. doi: 10.1039/c8nr07480a

13. Dong F, Chu W. Multichannel-independent information encoding with optical metasurfaces. Adv Mater. (2019) 31:1804921. doi: 10.1002/adma.201804921 beam. IL and HS guided the theoretical work. All authors analyzed and discussed the results.

\section{FUNDING}

This work was supported in part by the National Natural Science Foundation of China under Grant No. 11874270, the Fund Project for Shenzhen Fundamental Research Programme under Grant Nos. JCYJ20190808143409787 and JCYJ20180305125000525, and the China Scholarship Council under Grant No. 201908440615.

14. Zang X, Dong F, Yue F, Zhang C, Xu L, Song Z, et al. Polarization encoded color image embedded in a dielectric metasurface. Adv Mater. (2018) 30:1707499. doi: 10.1002/adma.201707499

15. Shao L, Zhu W, Leonov MY, Rukhlenko ID. Dielectric 2-bit coding metasurface for electromagnetic wave manipulation. J Appl Phys. (2019) 125:203101. doi: 10.1063/1.5094561

16. Lin L, Goh XM, McGuinness LP, Roberts A. Plasmonic lenses formed by two-dimensional nanometric cross-shaped aperture arrays for Fresnel-region focusing. Nano Lett. (2010) 10:1936-40. doi: 10.1021/nl1009712

17. Vashistha V, Vaidya G, Gruszecki P, Serebryannikov AE, Krawczyk M. Polarization tunable all-dielectric color filters based on cross-shaped Si nanoantennas. Sci Rep. (2017) 7:1-8. doi: 10.1038/s41598-017-07986-Z

18. Vashistha V, Vaidya G, Hegde RS, Serebryannikov AE, Bonod N, Krawczyk M. All-dielectric metasurfaces based on cross-shaped resonators for color pixels with extended gamut. ACS Photonics. (2017) 4:1076-82. doi: 10.1021/acsphotonics.6b00853

19. Saifullah Y, Waqas AB, Yang G-M, Xu F. Multi-bit dielectric coding metasurface for EM wave manipulation and anomalous reflection. Opt Express. (2020) 28:1139-49. doi: 10.1364/oe.383214

20. Zhang L, Zhang M, Liang $\mathrm{H}$. Realization of full control of a terahertz wave using flexible metasurfaces. Adv Opt Mater. (2017) 5:1700486. doi: 10.1002/adom.201700486

21. Akram MR, Mehmood MQ, Tauqeer T, Rana AS, Rukhlenko ID, Zhu W Highly efficient generation of Bessel beams with polarization insensitive metasurfaces. Opt Express. (2019) 27:9467-80. doi: 10.1364/oe.27.009467

22. Gerchberg RW. A practical algorithm for the determination of phase from image and diffraction plane pictures. Optik. (1972) 35:237-46.

Conflict of Interest: The authors declare that the research was conducted in the absence of any commercial or financial relationships that could be construed as a potential conflict of interest.

Copyright (c) $2020 \mathrm{Li}$, Ning, Zhang, Li, Su and Liang. This is an open-access article distributed under the terms of the Creative Commons Attribution License (CC BY). The use, distribution or reproduction in other forums is permitted, provided the original author(s) and the copyright owner(s) are credited and that the original publication in this journal is cited, in accordance with accepted academic practice. No use, distribution or reproduction is permitted which does not comply with these terms. 